$11-1-2016$

\title{
Fitting Flexible Parametric Regression Models with GLDreg in $\mathrm{R}$
}

Steve Su

Covance, allegro.su@gmail.com

Follow this and additional works at: http://digitalcommons.wayne.edu/jmasm

Part of the Applied Statistics Commons, Social and Behavioral Sciences Commons, and the Statistical Theory Commons

\section{Recommended Citation}

Su, Steve (2016) "Fitting Flexible Parametric Regression Models with GLDreg in R," Journal of Modern Applied Statistical Methods: Vol. 15 : Iss. 2 , Article 46.

DOI: $10.22237 /$ jmasm/1478004240

Available at: http://digitalcommons.wayne.edu/jmasm/vol15/iss2/46 


\section{Fitting Flexible Parametric Regression Models with GLDreg in $\mathbf{R}$}

\section{Steve Su}

Covance

Sydney, Australia

This article outlines the functionality of the GLDreg package in $\mathrm{R}$ which fits parametric regression models using generalized lambda distributions via maximum likelihood estimation and L moment matching. The main advantage of GLDreg is the provision of robust regression lines and smooth regression quantiles beyond the capabilities of existing known methods.

Keywords: $\quad$ Regression model, quantile regression, generalized lambda distributions, GLDreg, GLDEX, R

\section{Package GLDreq in $\mathrm{R}$}

The GLDreg package in $\mathrm{R}$ is designed to implement the Generalized Lambda Distribution (G $\mathrm{GD}$ ) regression model outlined in Su (2015) with some extensions. Currently, it is possible to fit $\mathrm{G} \lambda \mathrm{D}$ regression to data using maximum likelihood estimation (MLE) (Su, 2007a; b) and L moment matching (Asquith, 2007; Karvanen \& Nuutinen, 2008). Users may also chose initial values to start the model building process, or use the default searching algorithm using the ordinary least square regression model ( $\mathrm{Su}, 2015)$. The GLDreg package also allows user to fit quantile regressions parametrically and non-parametrically by: 1) fixing the intercept, 2) fixing coefficients other than the intercept, and 3) allowing all coefficients to vary.

The GLDreg package requires GLDEX (Su, 2007a; 2010). The GLDEX 2.0.0.1 package has a faster implementation of the G $\lambda \mathrm{D}$ fitting algorithm compared to its predecessors. This is because a number of frequently used codes have been written in C. In addition, the GLDEX 2.0.0.1 package has faster maximum likelihood fitting functions fun.RMFMKL.ml.m, fun.RPRS.ml.m for FKML

Steve Su is a Senior Statistician. Email him at: allegro.su@gmail.com. 


\section{STEVE SU}

(Freimer, Kollia, Mudholkar, \& Lin, 1988) and RS (Ramberg \& Schmeiser, 1974)

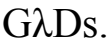

\section{Background}

Traditionally, the Pearson and Johnson systems are considered to be the standard approaches to identifying and fitting different types of statistical distributions to data. However, these systems require different mathematical functions to cover a range of symmetric and asymmetric distributions. Tukey's lambda distribution contains a class of symmetric distributions which can approximate a number of common distributions such as Normal and Cauchy distributions, and provide an indication as to whether heavy tailed distributions are needed. Ramberg and Schmeiser (1974) then generalized Tukey's lambda distribution to include both symmetric and asymmetric distributions, and this became known as the G $\lambda \mathrm{D}$. Although defined as a single mathematical function, G $\lambda \mathrm{D}$ can cover a broad range of statistical distributions which is much more efficient than the use of Pearson and Johnson systems involving several mathematical functions. Since then, the flexibility of G $\lambda \mathrm{D}$ has attracted a number of researchers. Today, along with the increased computation power and the introduction of dedicated packages for G $\lambda \mathrm{D}$ in $R$, it is now possible to fit G $\lambda D$ to data and extend the use of G $\lambda D$ in many areas of statistical analysis.

\section{Generalized Lambda Distributions}

The RS G $\lambda$ D (Ramberg \& Schmeiser, 1974) is defined by its inverse distribution function:

$$
\mathrm{F}^{-1}(u)=\lambda_{1}+\frac{u^{\lambda_{3}}-(1-u)^{\lambda_{4}}}{\lambda_{2}}, \quad 0 \leq u \leq 1
$$

From (1), $\lambda_{1}, \lambda_{2}, \lambda_{3}, \lambda_{4}$ are respectively the location, inverse scale, and shape parameters of generalized lambda distribution $\operatorname{G} \lambda \mathrm{D}\left(\lambda_{1}, \lambda_{2}, \lambda_{3}, \lambda_{4}\right)$. Note that $\lambda_{3}, \lambda_{4}$ are both shape parameters. Karian, Dudewicz, and McDonald (1996) noted that $\mathrm{G} \lambda \mathrm{D}$ is defined only if

$$
\frac{\lambda_{2}}{\lambda_{4}(1-u)^{\lambda_{4}-1}+\lambda_{3} u^{\lambda_{3}-1}} \geq 0
$$




\section{GLDreg IN R}

where $0 \leq \mathrm{u} \leq 1$.

Freimer et al. (1988) described another distribution known as FKML/FMKL $\mathrm{G} \lambda \mathrm{D}$. This distribution is slightly different to RS G $\lambda \mathrm{D}$ with respect to formulation. The FKML/FMKL G $\lambda \mathrm{D}$ is defined as:

$$
\mathrm{F}^{-1}(u)=\lambda_{1} \frac{\frac{u^{\lambda_{3}}-1}{\lambda_{3}}-\frac{(1-u)^{\lambda_{4}}-1}{\lambda_{4}}}{\lambda_{2}}, \quad 0 \leq u \leq 1
$$

Under (2), $\lambda_{1}, \lambda_{2}, \lambda_{3}, \lambda_{4}$ are respectively the location, scale, and shape parameters of the generalized lambda distribution. Again, both $\lambda_{3}, \lambda_{4}$ are shape parameters. Technically, the correct abbreviation for this distribution is FKML distribution, based on the correct ordering of authorship in the original paper. However, the use of the term FMKL distribution had been widespread in the literature and this package, along with GLDEX 2.0.0.1, will allow both " $f k m l$ " and "fmkl" specifications in the implementation of FKML distribution.

The fundamental motivation for the development of FKML G $\lambda D$ is that the distribution is defined over all $\lambda_{3}$ and $\lambda_{4}$ (Freimer et al., 1988). The only restriction on FKML G $\lambda \mathrm{D}$ is $\lambda_{2}>0$.

An extensive discussion of the shapes and properties of G $\lambda$ Ds can be found in the original papers (Ramberg \& Schmeiser, 1974; Freimer et al., 1988), as well as in subsequent works such as in $\mathrm{Su}(2015)$. Note the probability density functions of $\mathrm{G} \lambda \mathrm{Ds}$ is obtained by observing $\mathrm{F}^{-1}(u)=x$ and so

$$
\mathrm{f}(x)=\frac{1}{\frac{d \mathrm{~F}^{-1}(u)}{d u}}
$$

This is calculated using the Newton-Raphson method in GLDEX.

\section{Sketch of the Regression Fitting Mechanism}

The full fitting algorithm of G $\lambda \mathrm{D}$ regression models is provided in $\mathrm{Su}$ (2015). This section sketches the procedure in building these models. Consider the following regression model

$$
Y=X \beta+\varepsilon
$$




\section{STEVE SU}

The goal is to find estimated coefficients for $\beta$ under the condition $\varepsilon \sim \mathrm{G} \lambda \mathrm{D}\left(\lambda_{1}, \lambda_{2}, \lambda_{3}, \lambda_{4}\right)$, with the property such that $\mathrm{E}(\varepsilon)=0$. Consequently, the average deviation of actual and estimated values should be zero.

When deriving the probability density function of G $\lambda \mathrm{Ds}, \lambda_{1}$ will vanish, which means this parameter has no bearing on the overall shape of the distribution, only

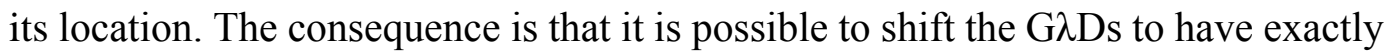
zero mean by calculating $\lambda_{1}$ after estimating $\lambda_{2}, \lambda_{3}, \lambda_{4}{ }^{1}$

The idea of fitting the G $\lambda \mathrm{D}$ regression in $\mathrm{Su}$ (2015) is to find a set of $\hat{\beta}$ by modelling the residuals using $\mathrm{G} \lambda \mathrm{D}$ through maximum likelihood estimation or $\mathrm{L}$ moment matching. The zero mean residual line is achieved by only allowing $\lambda_{2}, \lambda_{3}$, $\lambda_{4}$ to vary in the optimization process, and the intercept of the line is adjusted to ensure the residuals add up to zero. Statistical properties of G $\lambda \mathrm{D}$ regression coefficients are obtained by recreating actual values $y_{k}=\hat{y}_{k}+\hat{\varepsilon}_{k}$ for $k=1,2,3, \ldots$, $n$ observations by simulating $\hat{\varepsilon}_{k} \sim G \lambda D\left(\hat{\lambda}_{1}, \hat{\lambda}_{2}, \hat{\lambda}_{3}, \hat{\lambda}_{4}\right)$ and refitting the entire model and repeating the process, say 1000 times. This can all be done using the GLD.lm.full function and the goodness of fit of the model is assessed using the Kolmogorov-Smirnoff test and QQ plots. To run a simple model without any simulations, the function GLD.lm can be used instead.

Once a reference $G \lambda D$ regression line is found, quantile regression can be obtained by 1) fixing the intercept, 2) fixing coefficients other than the intercept, and 3) allowing all coefficients to vary. Case 1 and 3 are designed to fit non parallel lines for heteroskedastic data, and case 2 is primarily designed to fit parallel lines for homoskedastic data.

GLDreg allows non-parametric and parametric fitting of quantile regression lines. The non-parametric approach uses the least squares approach to find a $q$-th quantile $\mathrm{G} \lambda \mathrm{D}$ line such that the percentage of observations below the line corresponds to the $q$-th quantile. The parametric approach uses the least squares approach to find a $q$-th quantile $\mathrm{G} \lambda \mathrm{D}$ line such that the percentage of observations below the line (under a G $\lambda \mathrm{D}$ fit) corresponds to the $q$-th quantile. In the case of 1) and 3), the Nelder-Mead simplex algorithm from optim is used in the optimization process. In the case of 2), the Brent method from optim is used instead. The initial values for non-parametric quantile regression optimization are taken from the sample quantile of simulated regression coefficients obtained during the model building process. For the parametric approach, the initial values are taken from the non-parametric quantile regression. The estimates of both parametric and nonparametric $\mathrm{G} \lambda \mathrm{D}$ quantile regression can be obtained using a single wrapper function (GLD.quantreg). 


\section{GLDreg IN R}

\section{Using the Package}

GLDreg can be installed from $\mathrm{CRAN}^{2}$ through the R interface. Once installed, the following command will load the package.

> Library(GLDreg)

Loading required package: GLDEX

Loading required package: cluster

As usual, ?GLDreg will take user to the main help menu. Table 1 shows the main functions available under GLDreg.

Table 1. List of main functions

\begin{tabular}{|c|c|}
\hline Purpose & Function \\
\hline Fit GAD Regression only & G^D.Im \\
\hline $\begin{array}{r}\text { Fit GגD Regression and obtain statistical properties through } \\
\text { simulation }\end{array}$ & GLD.Im.full \\
\hline Fit $G \wedge D$ quantile regression & GLD.quantreg \\
\hline Plot summary graphics of $G \Lambda D$ regression & summaryGraphics.G $\lambda D . I m$ \\
\hline Plot quantile regression lines & fun.plot.q \\
\hline
\end{tabular}

Table 2. Fitting RS or FKML GAD regression using MLE or $L$ moment matching under GLD.Im or GLD.Im.full

\begin{tabular}{rrrr} 
Type of GAD & Type of estimation & Param & fun \\
\hline$R S$ & $M L E$ & "rs" & fun.RPRS.ml.m or fun.RPRS.ml \\
$R S$ & $L$ moment matching & "rs" & fun.RPRS.Im \\
FKML & $M L E$ & "fmkl" or "fkml" & fun.RMFMKL.ml.m or fun.RMFMKL.ml \\
FKML & $L$ moment matching & "fmkl" or "fkml" & fun.RMFMKL.Im \\
\hline
\end{tabular}

GLDreg currently implements MLE and L moment matching for G $\lambda \mathrm{D}$ regression. The associated param and fun inputs for GLD.lm and GLD.lm.full are given in Table 2. The fun.RPRS.ml.m and fun.RMFMKL.ml.m functions are faster implementations of MLE than the previous fun.RPRS.ml and fun.RMFMKL.ml functions. 


\section{STEVE SU}

\section{Engel Dataset}

This example uses the well-known Engel dataset from the quantreg package in R. The following fits a full FKML G $\lambda D$ regression model using MLE, along with simulations (default is 1000 runs), and then fits a quantile regression for quantiles from 0.1 to 0.9 in 9 equal spacings with fixed intercept and varying slopes.

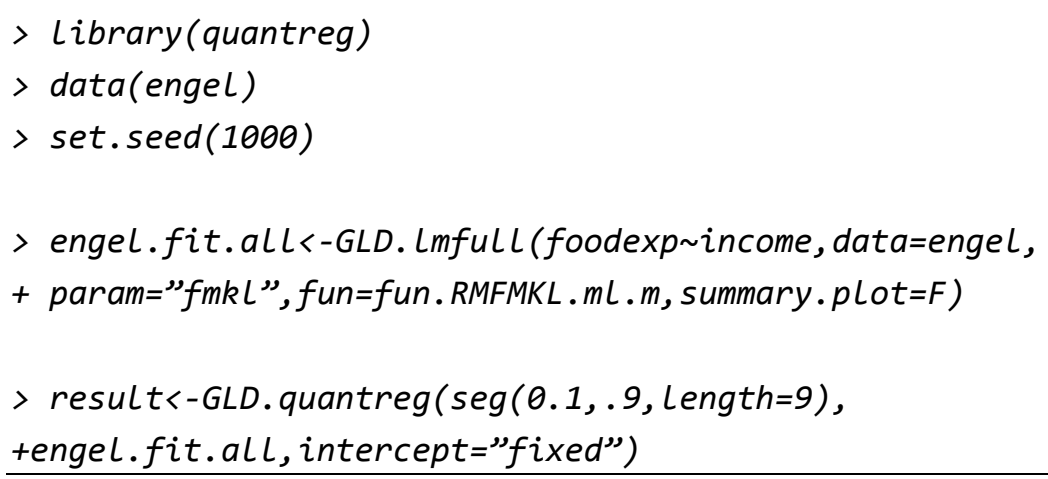

There are warnings associated with using the Nelder-Mead algorithm for single parameter optimization problems. To check whether optimization has indeed been achieved for quantile regression, the easiest way is to check whether the proportion of the fitted quantile line below the response variable corresponds to the level of quantile specified. The multiplication of 100 is to convert the quantiles into percentages, and the aim is to see how close these are to 10, 20, 30, 40, 50, 60, 70, 80 and 90.

$>\operatorname{sapply}(1: 9$, function $(i) \operatorname{sum}($ engel $\$$ foodexp-
cbind $(1$, engel $\$$ income $) \% * \%(\operatorname{result}[1: 2, i])<\theta) /$ nrow $($ engel $) * 100)$

[1] $8.085106 \quad 22.127660 \quad 30.638298 \quad 38.723404 \quad 51.489362 \quad 58.723404$

67.65957482 .97872390 .638298

The results are reasonably close to the intended quantiles. Note differences occur because the optimization here is based on matching the quantile of fitted G $\lambda \mathrm{D}$ rather than the empirical data, the idea being that if the fitted G $\lambda \mathrm{D}$ is close to the actual distribution, the quantile lines obtained here will be more robust to changes in empirical data.

Alternatively, quantile lines could be obtained using the non-parametric approach using the following, and checked whether the desired quantile line has 


\section{GLDreg IN R}

been achieved despite warnings from the optim function. Obviously, the match is better, simply because optimization was done based on empirical data.

Once the quantile lines are obtained, it is possible to plot them; in this case, the parametric G $\lambda \mathrm{D}$ quantile lines are plotted in Figure 1.

$>$ fun.plot. $q(x=$ engel $\$$ income, $y=e n g e l \$$ foodexp, fit=engel.fit.all[ $[1]]$, result)

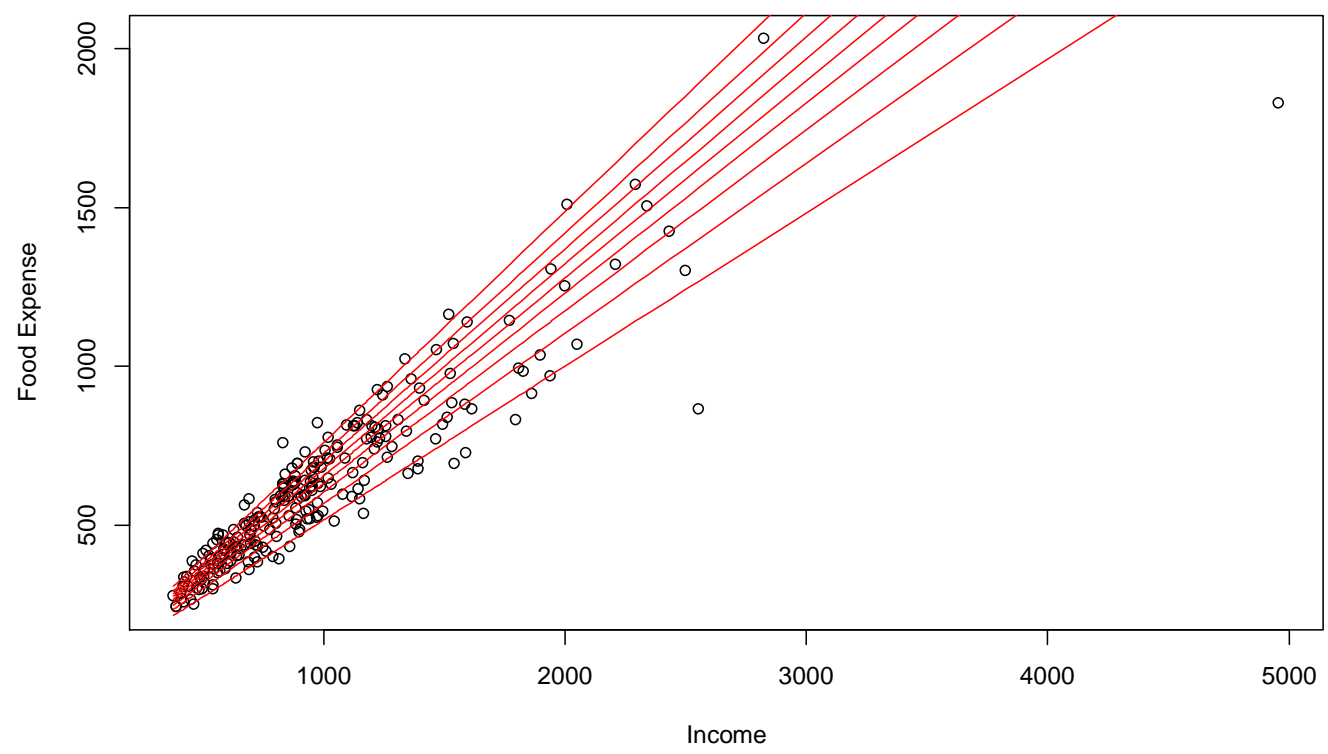

Figure 1. FKML $\mathrm{G} \lambda \mathrm{D}$ quantile regression for Engel dataset

It is also possible to speed up GLD.lm.full by reducing the number of simulations to 100 by setting $n=100$, if the primary purpose is to obtain quantile regression rather than looking at the statistical properties of the coefficients. GLDreg also provides a graphic summary of regression coefficients based on object obtained from GLD.lm.full, using the summaryGraphics.gld.lm function, and the graphics are shown in Figure 2 and Figure 3. 


\section{STEVE SU}

summaryGraphics.gld.Lm(engel.fit.all)

Shown in Figure 2 is the statistical distribution of coefficients obtained using simulation, and the $95 \%$ interval is obtained directly from simulated results. Shown in Figure 3 is how well FKML G $\lambda \mathrm{D}$ fits the data; in this case the fit is quite good, with Kolmogorov-Smirnoff goodness of fit $p$-value exceeding 5\% and the QQ plot indicating a close fit.

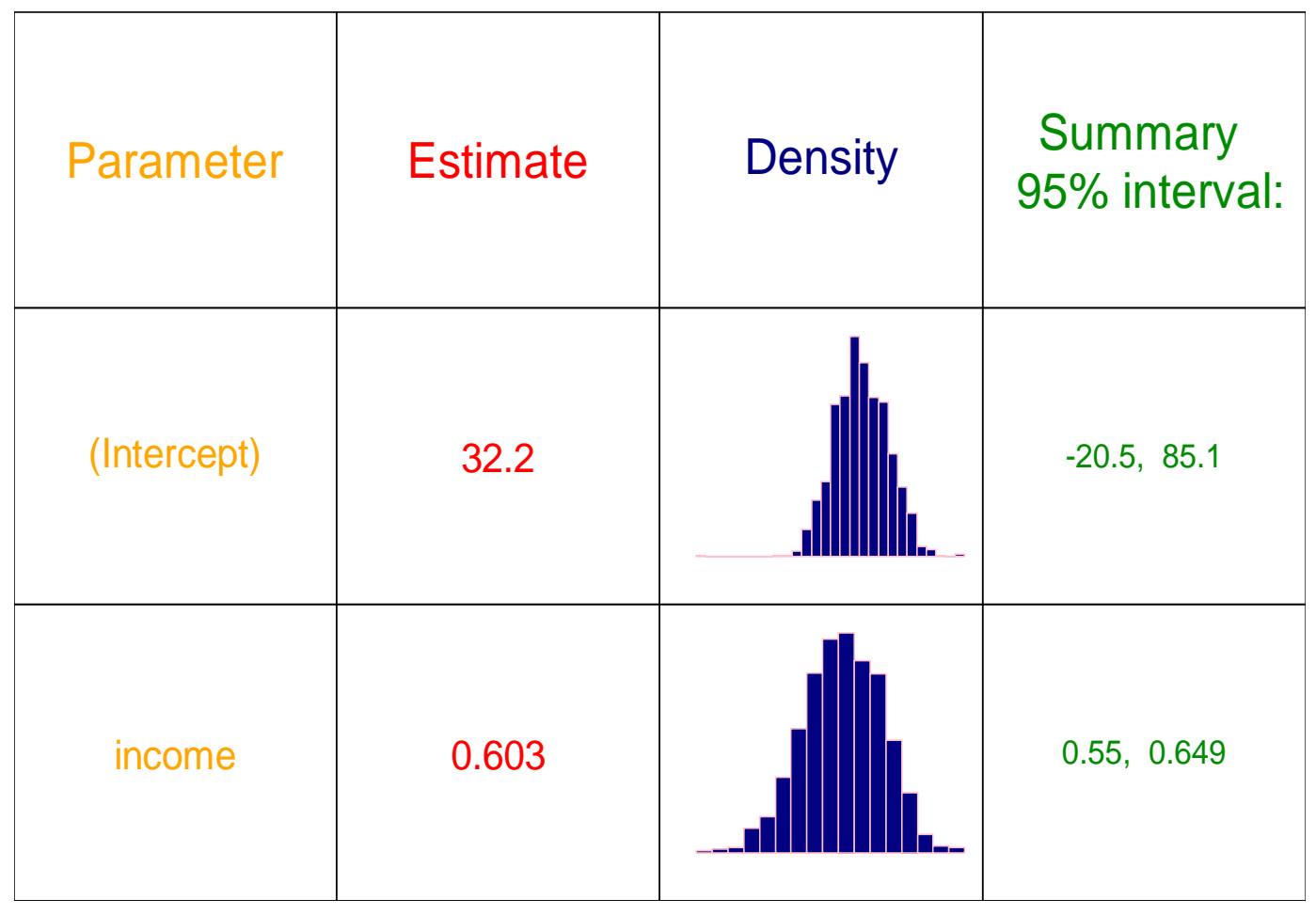

Figure 2. FKML GAD quantile regression for Engel dataset coefficient summary plot 


\section{GLDreg IN R}

QQ plot for residuals Version 1

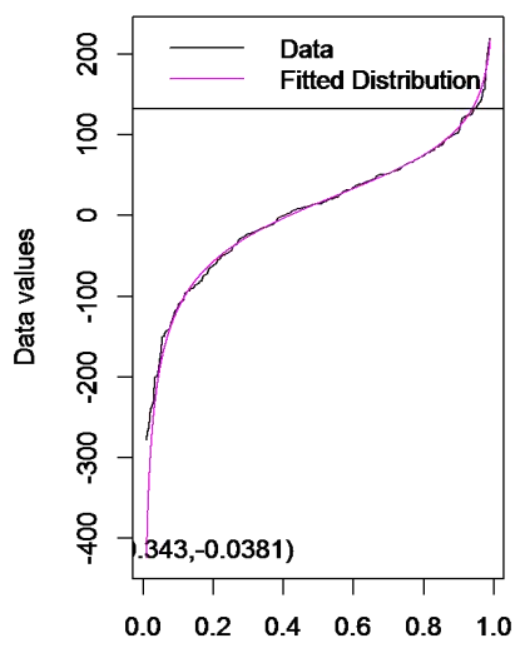

QQ plot for residuals Version 2

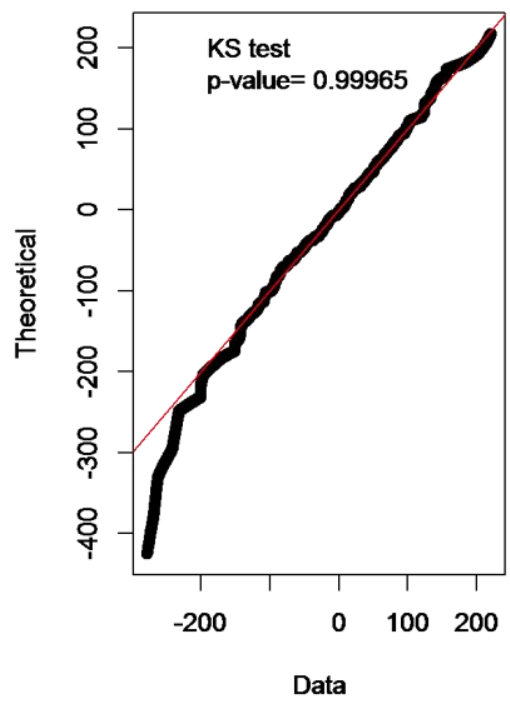

Figure 3. FKML GAD quantile regression for Engel dataset $-Q Q$ plots

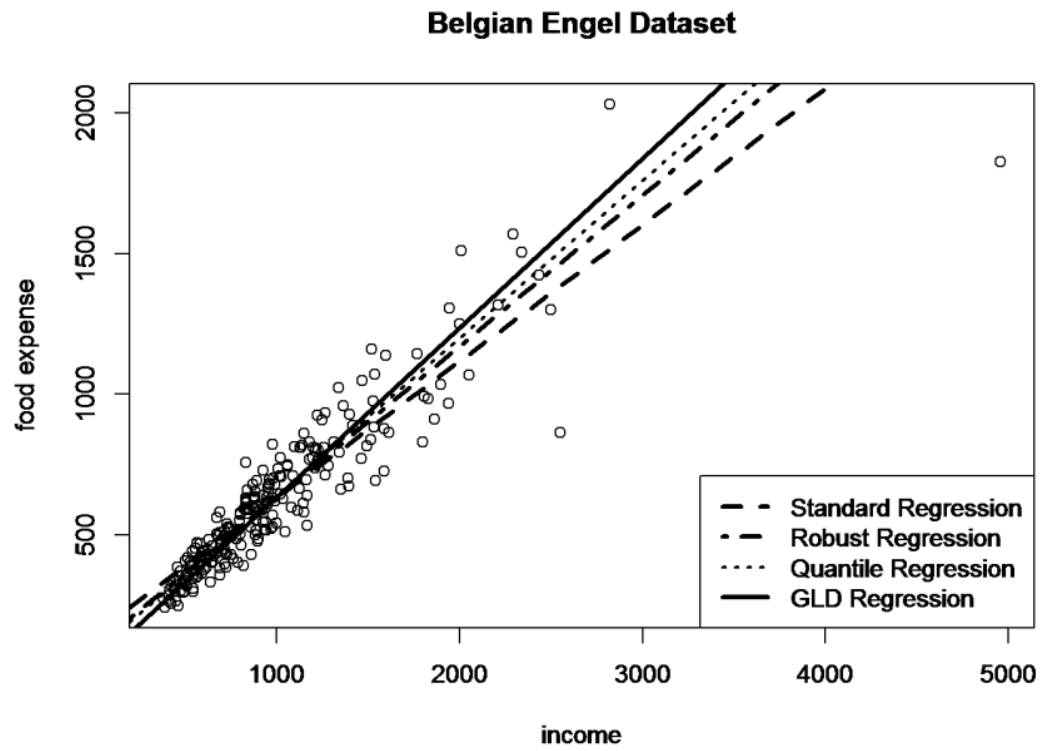

Figure 4. Comparison of different regression techniques for Engel dataset 


\section{STEVE SU}

Note the FKML G $\lambda \mathrm{D}$ regression line is the most robust to outliers compared to linear regression, robust regression, and quantile regression at median. This can be seen using the following code, which is displayed in Figure 4.

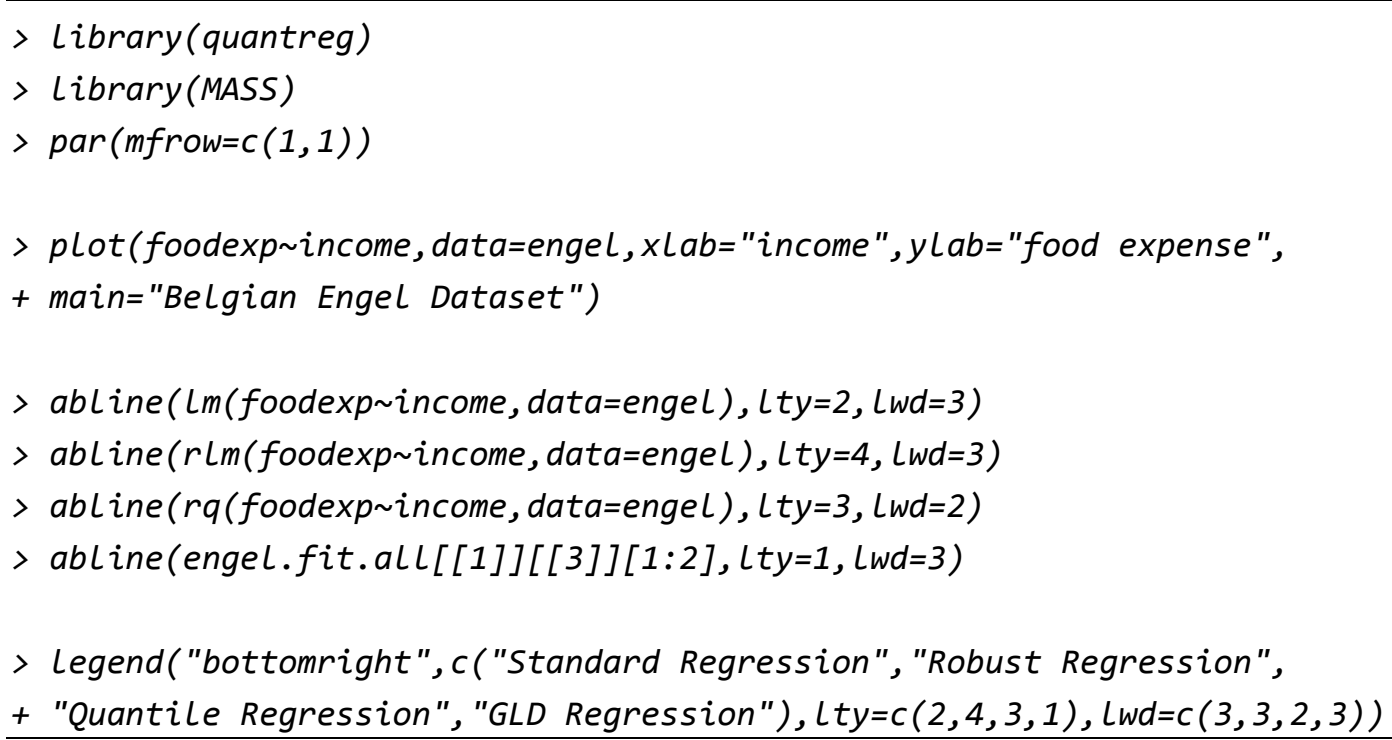

\section{Simulated Motorcycle Accident Dataset}

The mcycle dataset is a simulated motorcycle accident dataset from MASS library in R. It is possible to fit splines to this dataset, and a reasonable strategy is to fit time before 15 seconds separately as was done in $\mathrm{Su}$ (2015). In the same fashion as in Engel dataset analysis, the full G $\lambda \mathrm{D}$ regression is fitted first, followed by evaluation of quantile regression lines, for which parallel lines appear to be quite suitable for this dataset. The only difference is that the modelling is split into two parts. In this case, the first part (before 15 seconds) was fitted using splines with 8 degrees of freedom using RS G $\lambda \mathrm{D}$ via maximum likelihood estimation. The second part (greater or equal to 15 seconds) was fitted using splines with 15 degrees of freedom using FKML G $\lambda \mathrm{D}$ via maximum likelihood estimation. The code to produce Figure 5 is given below.

\footnotetext{
> Library(MASS)

$>$ Library(splines)

$>$ cutoff $<-15$

$>$ mcycle.part1<-mcycle[mcycle\$times<cutoff, ]
} 


\section{GLDreg IN R}
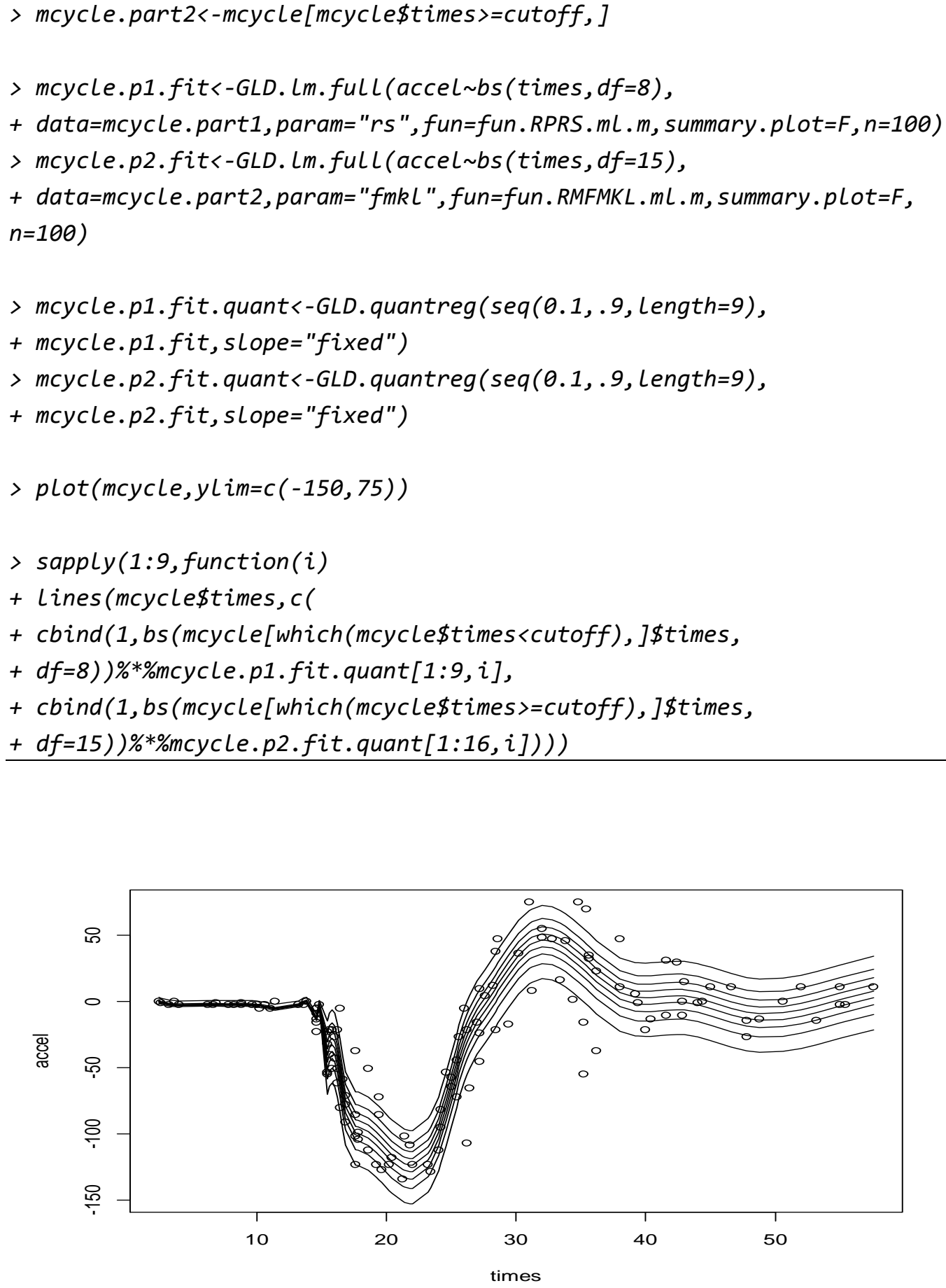

Figure 5. Simulated motorcycle accident dataset 


\section{STEVE SU}

\section{Modified Crime Data}

A fair exposition of $\mathrm{G} \lambda \mathrm{D}$ regression needs to include a discussion of its weaknesses. Being a regression model involving numerical computations, the initial values play an important role in deriving the final model results. While all numerical optimization problems suffer from bad choice of initial values and it is a general limitation of the Nelder-Mead optimization method, it is possible to still attain the attractive robust property of G $\mathrm{DD}$ regression by simply choosing a better set of initial values.

This example used the crime dataset from UCLA website ${ }^{3}$ and examined the relationship between crime rate and percentage of single family parents. To examine the behavior of regression models under extreme outliers, two data points were altered in this illustration. The coding involved in extracting and altering the dataset is given below.

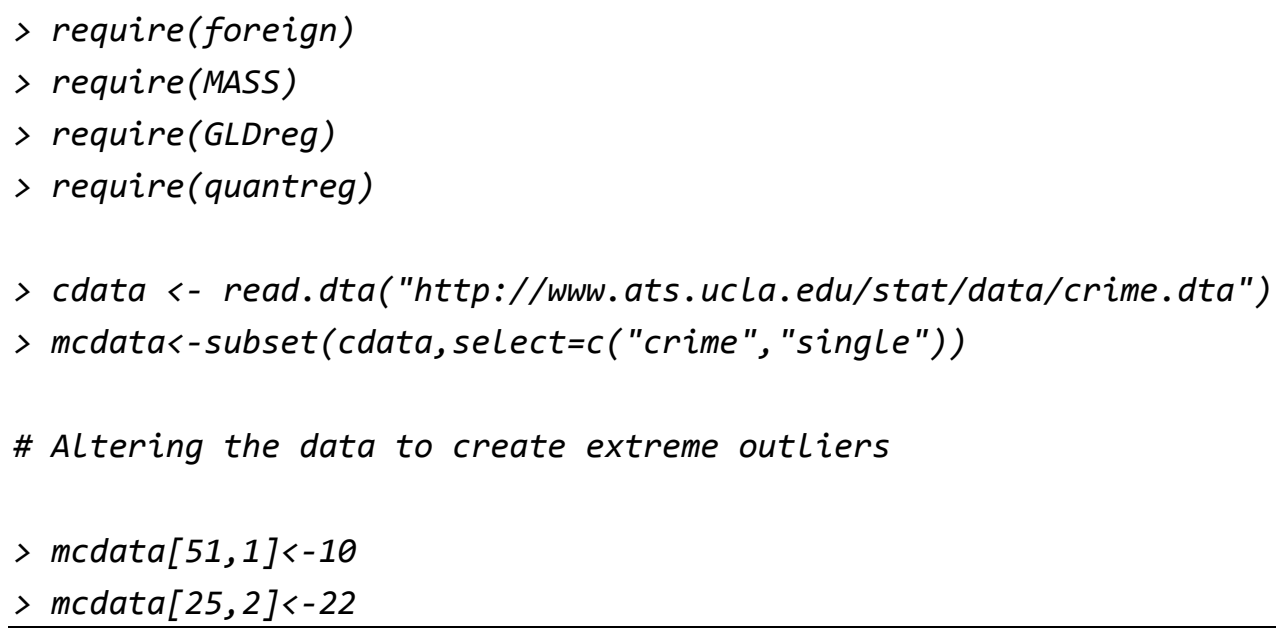

If the modeler begins with RS G $\lambda \mathrm{D}$ using MLE using the default initial values, the resulting G $\mathrm{GD}$ regression line is shown in panel A of Figure 6, which is perhaps the worst model among all others (linear regression, robust regression, and quantile regression). This happens because the default initial values was based on linear regression and a less-than-optimal line was found using the Nelder-Mead simplex algorithm.

\footnotetext{
$>\operatorname{par}(m f r o w=c(2,2))$

$>$ plot(crimensingle, data=mcdata, ylab="Violent crimes per 100,000 people",
} 


\section{GLDreg IN R}

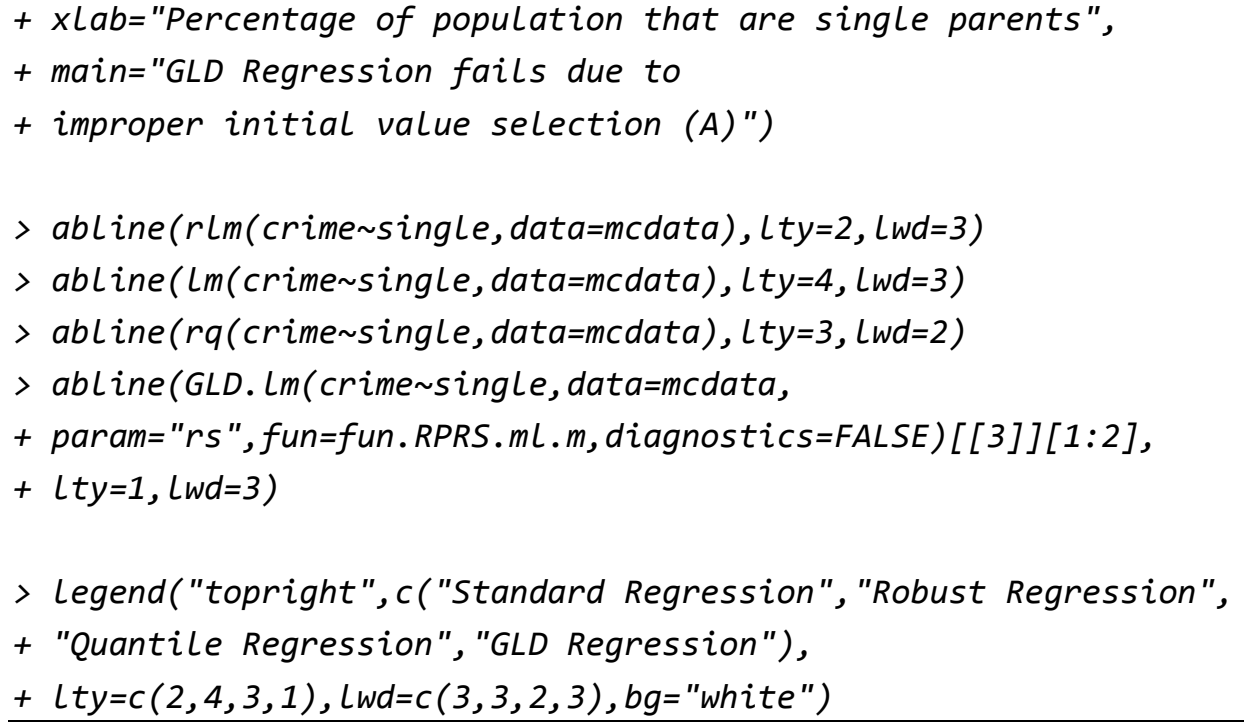

It is possible to improve the quality of fit by using different initial values. In the following, init1 uses quantile regression coefficients, init2 uses robust regression coefficients, and init3 uses G $\lambda \mathrm{D}$ regression coefficients obtained by removing the outliers at the bottom right corner of the graph.

$>$ init1<-rq(crime single, data=mcdata)\$coeff

$>$ init2<-rlm(crime single, data=mcdata)\$coeff

$>$ init3<-GLD. Lm(crime single,

+ data=rbind (mcdata[1:24, ], mcdata[26:50,]),

+ param="rs",fun=fun.RPRS.mL.m, diagnostics=FALSE) [[3]][1:2]

The modeler can then refit $\mathrm{RS}$ G $\lambda \mathrm{D}$ regression model using maximum likelihood estimation by using these initial values using the following code.

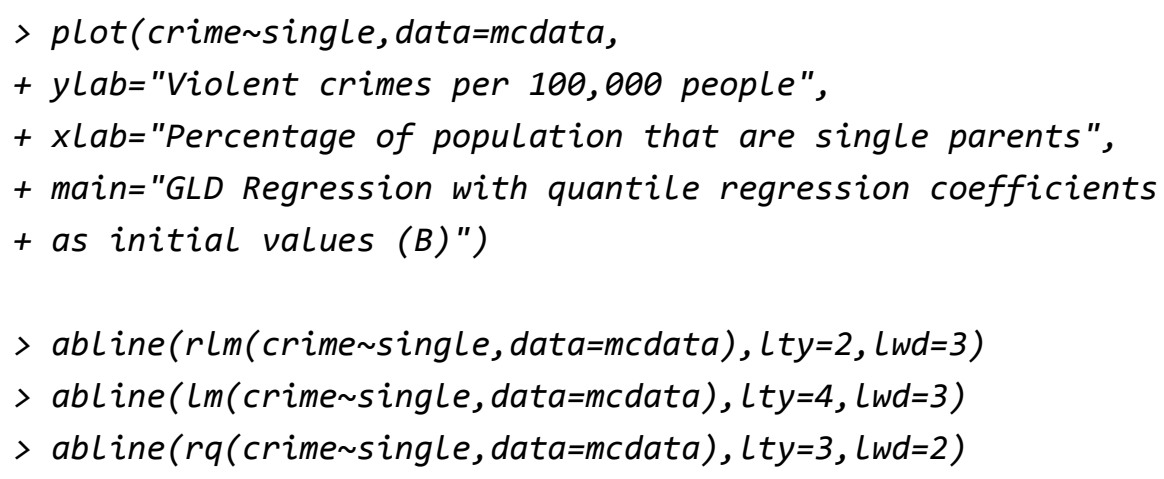




\section{STEVE SU}

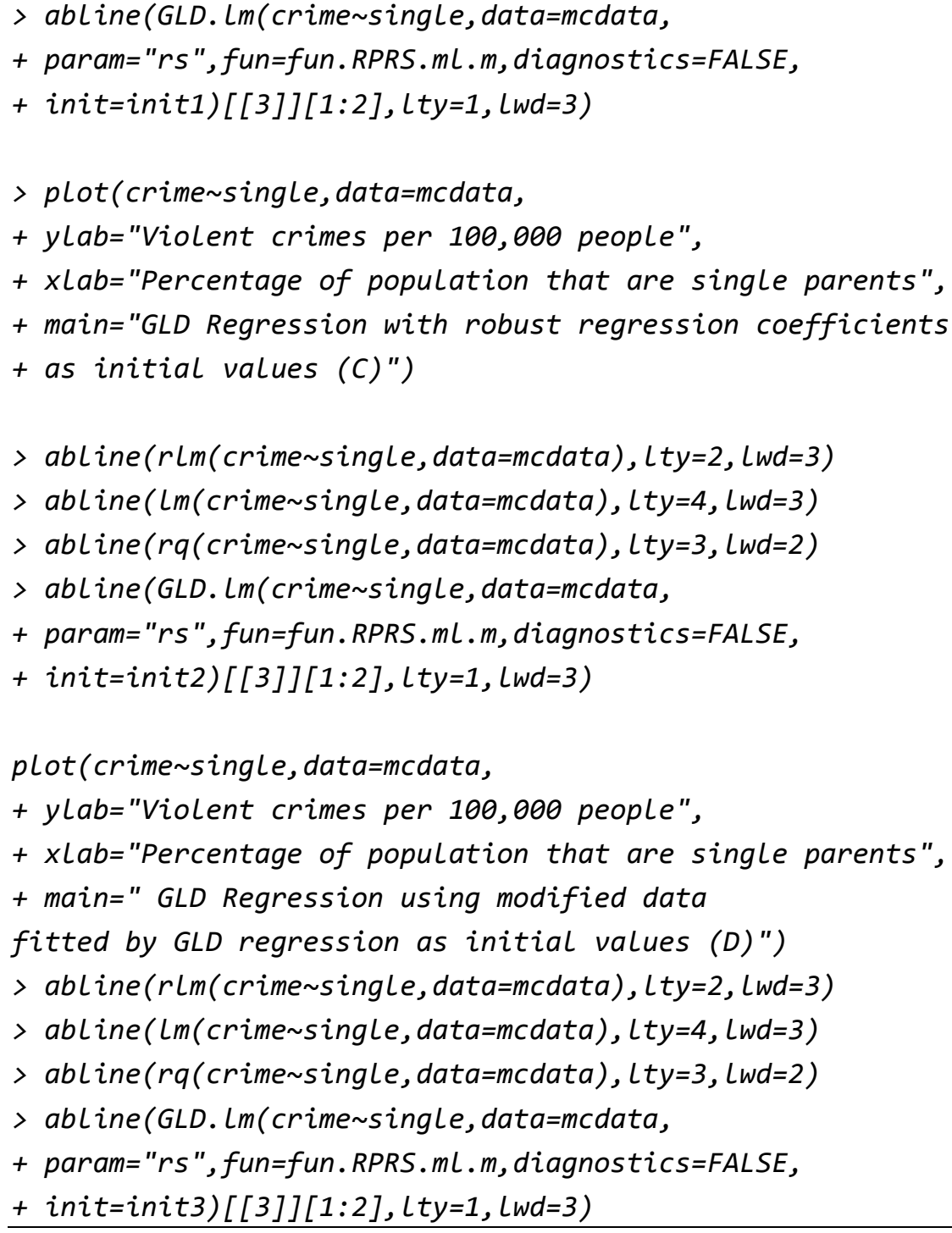

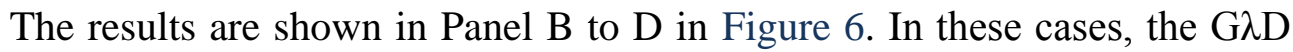
regression line is now the most robust among all regression lines. The importance of checking the resulting fit and trying out different sets of initial values for optimization is highlighted in this example. 

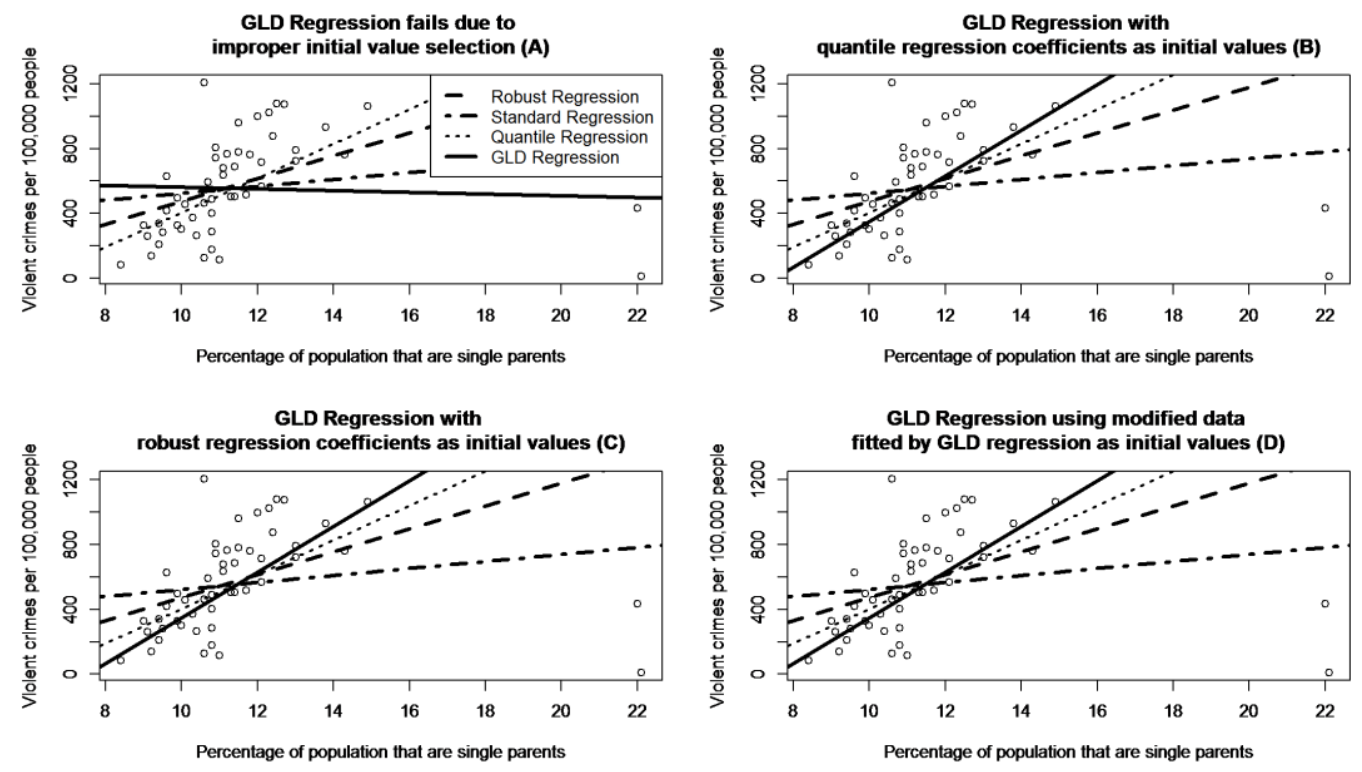

Figure 6. Modified crime data set

\section{Occupation Dataset}

The last example involves modelling beyond a single explanatory variable on the Duncan dataset from car in R. In this case, a FKML G $\lambda$ D regression using $L$ moment estimation was fitted to the dataset with initial values taken from robust linear regression. It is worthwhile to plot the fitted values against actual values, and to see whether the observations correspond to a 45 degree line from the origin. Large deviations from a linear trend would suggest the model does not fit well with respect to the response variable. The coding to carry the above tasks is given below.

The resulting fit is similar to the robust regression result, and QQ plots and high $p$-value (Figure 7) suggest the G $\lambda \mathrm{D}$ regression fit is a good one. Statistical properties obtained (Figure 8) suggest that the most important variable related to income is prestige. Figure 9 shows that, while the fitted values are not extremely accurate with respect to actual values, the general linear trend is still observed, indicating that the linear form of the model is appropriate.

As a final remark, it can be worthwhile to fit quantile regression lines for fitted values against actual values to give a range of likely actual values that could be obtained using the linear model.

$>$ Library(MASS) 


\section{STEVE SU}
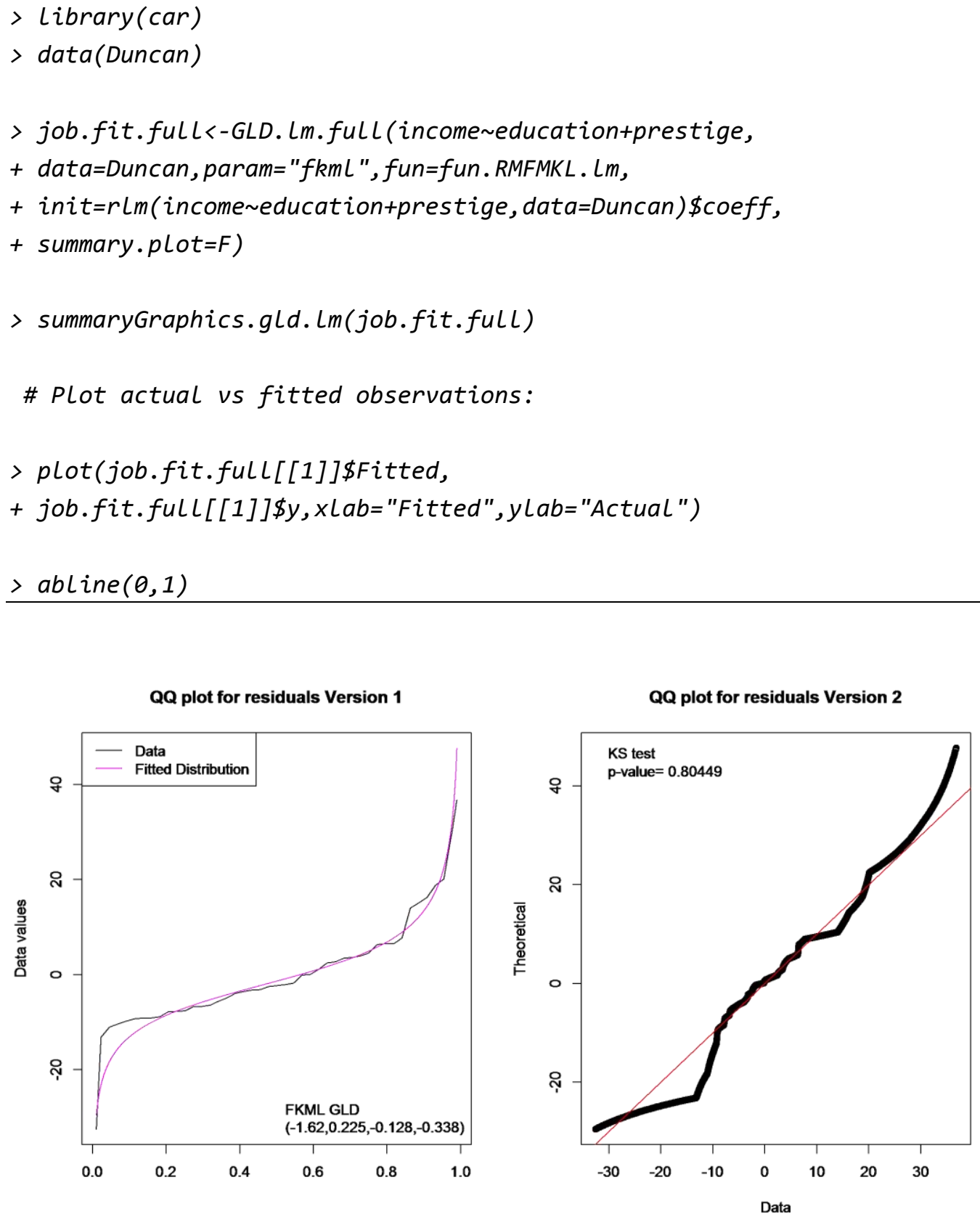

Figure 7. Occupation data set modelling $-\mathrm{QQ}$ plots 


\section{GLDreg IN R}

\begin{tabular}{|c|c|c|c|}
\hline Parameter & Estimate & Density & $\begin{array}{l}\text { Summary } \\
95 \% \text { interval: }\end{array}$ \\
\hline (Intercept) & 7.69 & & $-3.07,19.8$ \\
\hline education & 0.109 & & $-0.13,0.337$ \\
\hline prestige & 0.597 & & $0.403,0.841$ \\
\hline
\end{tabular}

Figure 8. Occupation data set modelling - summary plots

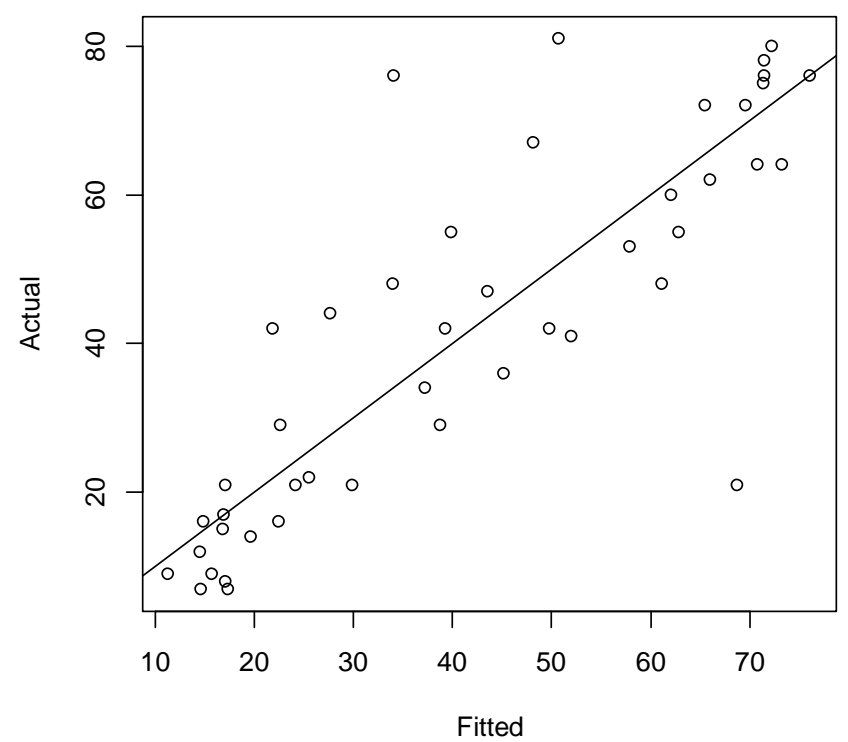

Figure 9. Occupation data set modelling - actual vs. fitted values 


\section{STEVE SU}

\section{Conclusion}

The flexibility of $\mathrm{G} \lambda \mathrm{D}$ regression models was illustrated; rather than confining the regression model to only examining the mean or median as is the case of linear regression or classic quantile regression model, the G $\lambda D$ regression models attempt to fit a line that represent a typical value for the dataset which may or may not correspond to standard measures such as the mean or median. Some extensions were also shown beyond the methodology described in $\mathrm{Su}$ (2015), and two different optimization schemes (L moment matching and maximum likelihood estimation) were implemented to increase the versatility of $\mathrm{G} \lambda \mathrm{D}$ regression in different modelling situations. Potential shortcomings of $G \lambda D$ regression in initial value selection were illustrated, as well as how different initial values could lead to better regression fits, which is a problem for many model fitting problems involving numerical computations. Since its inception, G $\lambda$ Ds have been used to model a wide range of empirical data, and the flexibility and robustness of G $\lambda \mathrm{D}$ regression is particularly attractive either as a check for standard results or as a replacement. It is hoped that the introduction of this work would encourage future researchers to develop new methodological improvements to further enhance the usability of G DDs in practice.

\section{References}

Asquith, W. (2007). L-moments and TL-moments of the generalized lambda distribution. Computational Statistics and Data Analysis, 51(9), 4484-4496. doi: 10.1016/j.csda.2006.07.016

Freimer, M., Kollia, G., Mudholkar, G. S., \& Lin, C. T. (1988). A Study of the generalized Tukey lambda family. Communications in Statistics - Theory and Methods, 17(10), 3547-3567. doi: 10.1080/03610928808829820

Karian, Z., Dudewicz, E., \& McDonald, P. (1996). The extended generalized lambda distribution systems for fitting distributions to data: history, completion of theory, tables, applications, the "final word" on moment fits.

Communications in Statistics - Computation and Simulation, 25(3), 611-642. doi: 10.1080/03610919608813333

Karvanen, J., \& Nuutinen, A. (2008). Characterizing the generalized lambda distribution by L-moments. Computational Statistics and Data Analysis, 52(4), 1971-1983. doi: 10.1016/j.csda.2007.06.021 


\section{GLDreg IN R}

Ramberg, J., \& Schmeiser, B. (1974). An approximate method for generating asymmetric random variables. Communications of the Association for Computing Machinery, 17(2), 78-82. doi: 10.1145/360827.360840

$\mathrm{Su}, \mathrm{S}$. (2007a). Fitting single and mixture of generalized lambda distributions to data via discretized and maximum likelihood methods: GLDEX in R. Journal of Statistical Software, 21(9), 1-17. doi: 10.18637/jss.v021.i09

Su, S. (2007b). Numerical maximum log likelihood estimation for generalized lambda distributions. Computational Statistics and Data Analysis, 51(8), 3983-3998. doi: 10.1016/j.csda.2006.06.008

Su, S. (2010). Chapter 15: Fitting GLD to data using the GLDEX 1.0.4 in R. In Z. Karian \& E. Dudewicz (Eds.), Handbook of distribution fitting methods with $R$ (585-608). Boca Raton: CRC Press/Taylor \& Francis.

$\mathrm{Su}$, S. (2015). Flexible parametric quantile regression model. Statistics and Computing, 25(3), 635-650. doi: 10.1007/s11222-014-9457-1 


\section{STEVE SU}

\section{Footnotes}

1. The theoretical mean for RS G $\lambda \mathrm{D}$ is

$$
\lambda_{1}+\frac{\frac{1}{\lambda_{3}+1}-\frac{1}{\lambda_{4}+1}}{\lambda_{2}}
$$

and the theoretical mean for FKML G $\lambda \mathrm{D}$ is

$$
\lambda_{1}-\frac{\frac{1}{\lambda_{3}+1}-\frac{1}{\lambda_{4}+4}}{\lambda_{2}}
$$

2. CRAN can be accessed at http://cran.r-project.org/

3. The UCLA crime dataset can be accessed at http://www.ats.ucla.edu/stat/data/crime.dta 